\title{
Mapping with expression
}

DOI:
In recent years, several studies have made inroads into the genetic control of gene expression, showing that transcript abundance for individual genes can be treated as heritable quantitative traits. Although this work promises to improve our understanding of gene regulation, the published work has been limited by relatively small sample sizes, incomplete coverage of the genome, and the use of transformed cells. Three new papers address these shortcomings in a complementary fashion, and provide a more complete picture of so-called 'expression QTLs' (eQTLs).

Although the use of cell lines has obvious advantages, it is also possible that transcription in such cells does not represent the natural state of gene expression. Harald Göring and colleagues addressed this potential problem by carrying out genomewide transcriptional profiling of freshly isolated lymphocytes from more than 1,200 participants in the San Antonio Family Heart Study (previous eQTL studies had typically been done in 100-200 individuals). They confirmed the broad, if modest, heritability of most transcript levels, and proceeded to carry out linkage analysis of all transcripts with 432 highly polymorphic microsatellite markers. More than 1,300 cis-regulated transcripts were identified, with the strength of cis-regulation correlating with transcript heritability. Correlations between cis-regulated transcripts and high-density lipoprotein (HDL) cholesterol levels led to the identification of a novel candidate gene, vanin 1

(VNN1) for heart disease.

Although the study by Göring et al. examines a large number of individuals, the use of a relatively small set of microsatellite markers limits the mapping resolution of the individual eQTLs. By contrast, the two other new studies use genome-wide SNP association. In the study by William Cookson and colleagues, transcript levels from more than 20,000 genes in lymphoblastoid cell lines were associated with more than 400,000 SNPs that had been genotyped in 400 children as part of a study of asthma. In addition to generating a rich resource of SNPs associated with transcript abundance, available at the downloadable mRNA by SNP Browser, this effort also shows how such a resource can be leveraged to pinpoint variants that might be involved in asthma as well as other diseases. For example, a 206-kb susceptibility locus for asthma was found to harbour highly associated SNPs that were also associated with expression levels of ORM1-like 3 (ORMDL3; see Further Reading), suggesting that cis-regulatory variation in this gene is associated with risk of asthma.

Finally, Emmanouil Dermitzakis and colleagues carried out a genome-wide association study of gene expression using 2.2 million SNPs derived from phase II of the International HapMap Project, and transcriptional profiles of lymphoblastoid cell lines derived from all
270 participants in the HapMap. Their results show not only a large number of cis associations of gene expression with particular SNPs, but also confirm previous findings on significant differences between populations. Finally, the density of genotyped SNPs allowed the authors to map many of the associations to regions very close to the transcriptional start sites of genes, in regions of highly conserved sequence.

All told, these studies provide the most comprehensive view yet of the abundant cis-regulatory variation in the human genome. In concert with genome-wide association studies of disease, they should provide powerful aids to understanding how disease susceptibility varies among individuals.

Alan Packer, Senior Editor Nature Genetics

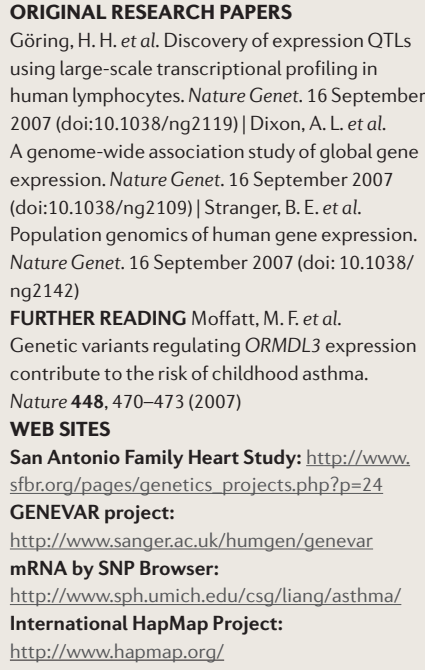

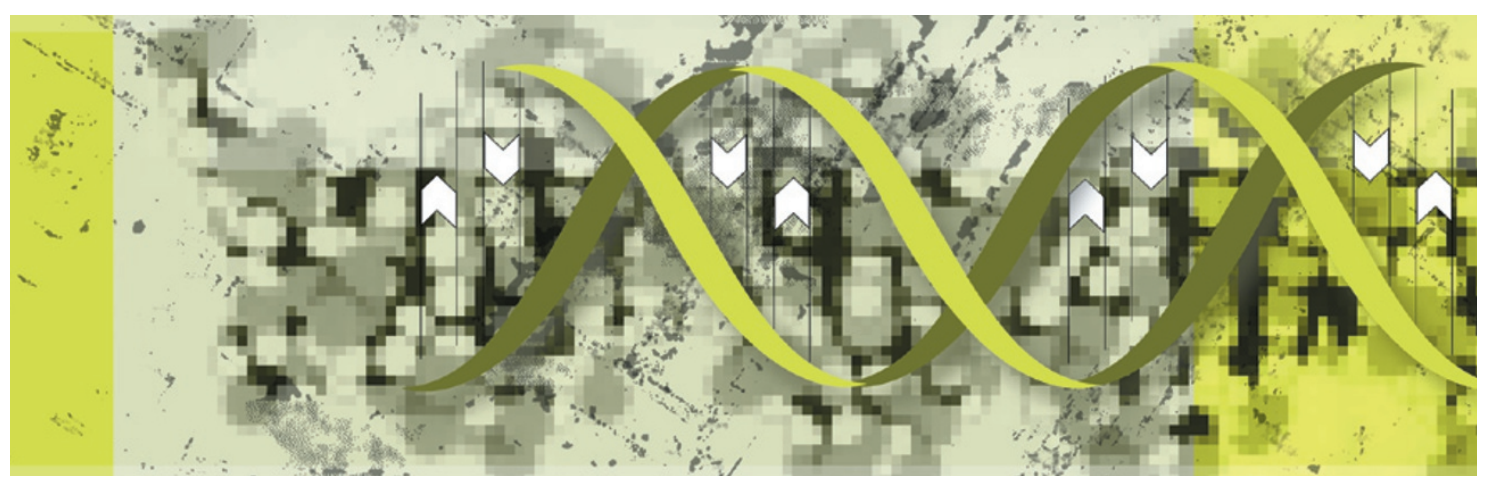

